\title{
Numerical and Experimental Research on the Influence of the Centrifugal Pumps Rotor Damage on Their Natural Frequency Response
}

Marek Dudziński ${ }^{1}$, Andrzej Grządziela ${ }^{1}$, Marcin Kluczyk $^{1}$, Janusz Musiał $^{2}$, Łukasz Muślewski $^{2}$ and Michał Pająk ${ }^{3}$ 1. Mechanical-Electrical Faculty, Polish Naval Academy, Gdynia 81103, Poland

2. Machine Maintenance Department, University of Technology and Life Science, Bydgoszcz 85225, Poland

3. Faculty of Mechanical Engineering Radom, University of Technology, Radom 26600, Poland

\begin{abstract}
The paper presents process of creating a centrifugal pump rotor model in CAD environment. Modeling of a virtual object was divided into two stages, modeling of the efficient pump and the simulated failure of one of the impeller's blades. Comparison of the results of the resonance frequency obtained from the model analysis, with those obtained from measurements on the actual object was shown. Measurements and simulations were conducted on the pump before and after the simulated damaged of the rotor. In order to verify the model the rotor of pump was weighted and compared with the masses of the respective components obtained from the virtual object. In the second stage genuine rotor was subjected to the experimental modal analysis.
\end{abstract}

Key words: Centrifugal pump, experimental modal analysis, numerical calculations.

\section{Introduction}

The object of study was rotor derived from the pump SKA.4.02. It is a centrifugal pump, circulatory with side ring channel and impeller of open type. The main advantage of this type of pump is their ability of self-suction, on the other hand like all centrifugal pumps they are exposed to a high risk of cavitation. The phenomenon of cavitation erosion can quickly cause damage to the pump components, including the rotor. The resulting damage can cause changes in amplitude-frequency characteristics of the pump. Under certain circumstances it could lead to its work in the field of the resonance frequency. Increasing rotor vibration energy transferred by bearings to cast iron hull can cause it to crack.

\section{Materials}

Creating the model had been started from the initial

Corresponding author: Andrzej Grządziela, Ph.D., ass. prof., research field: machine vibrations, underwater explosions. E-mail: a.grzadziela@amw.gdynia.pl. calculations of the resonance frequency of a propeller single blade. To obtain results the analytical method of calculations had been used. Calculations were conducted in two cases: firstly in normal geometrical dimensions of blade, secondly for blade with decreased height with simulated damage of the blade. For calculations the following dimensions had been used:

- blade efficient: thickness $-0.005 \mathrm{~m}$

$$
\begin{aligned}
& \text { width }-0.025 \mathrm{~m} \\
& \text { height }-0.06 \mathrm{~m} .
\end{aligned}
$$

- blade damaged: thickness $-0.005 \mathrm{~m}$

$$
\begin{aligned}
& \text { width }-0.025 \mathrm{~m} \\
& \text { height }-0.05 \mathrm{~m} . \\
& \text { Material data: }
\end{aligned}
$$

Brass: $E=115 \cdot 10^{9}[\mathrm{~Pa}] \rho=8500\left[\frac{\mathrm{kg}}{\mathrm{m}^{3}}\right]$.

Calculations of natural vibration frequency (for flexural vibration) are determined from the Eq. (1) [1]:

$$
\omega_{n}=\left(\lambda_{n} l\right)^{2} \sqrt{\frac{E I}{\rho A l^{4}}}
$$


For $\mathrm{n}=1 \quad \lambda_{n} l=1.875$

$\lambda —$ slenderness ratio calculated from the Eq. (2):

$$
\lambda=\frac{l_{w}}{i_{\min }}
$$

where: $l_{w}=\mu l$.

Assuming $\mu=2$ for blade one sided fixed.

The smallest radius of inertia was calculated using the Eq. (3):

$$
i_{\min }=\sqrt{\frac{I_{\min }}{A}}
$$

where:

$i_{\text {min }}$ - the smallest main central moment of inertia,

$A-$ cross-sectional area.

After substitution data patterns into the formulas results was obtained. The results were shown in Table 1, which also provided the results of computer simulation in CAD. Simulations were also presented in Figs. 1 and 2.

In the case of shortening the blade from $0.06 \mathrm{~mm}$ to $0.05 \mathrm{~mm}$ (17\% weight loss) as far as other dimensions was the same the first natural frequency increased 1.44 fold.

\subsection{CAD calculations}

The geometry of the rotor presented on Fig. 3 was discretized by 15,999 tetragonal linear elements, it allows to obtain 26,918 nodes resulting in a model shown in Figs. 4 and 5.

Assignment material was brass with a density of $8,470 \mathrm{~kg} / \mathrm{m}^{3}$. For the verification of the model mass, the rotor was weighed and compared with the results obtained from real object. The weight of a real object is $1,880 \mathrm{~kg}$ while the virtual model received mass was $1,884 \mathrm{~kg}$. Due to the significant impact of mass distance from the axis of rotation to the mass moment of inertia, particular attention was given to map the shape of the blades, especially the elements lying farthest from the axis of rotation [2]. It can be considered that the model was made with a small error of $0.004 \mathrm{~kg}$ which corresponds to the error of $0.21 \%$. Object was analyzed by creating a pin bond which allows the rotor free circulation (Fig. 5).

Numerical calculations were repeated for the damaged model through shortened by $0.03 \mathrm{~m}$ blade, and when it is completely cut out. The resultant resonance frequencies were shown in Table 2.

\subsection{Experimental Modal Analysis}

Experimental modal analysis (EMA) is the process of experimental determination of the resonance frequency of the test system. Using appropriate techniques, it is also possible to determine the mode

\begin{tabular}{|c|c|c|c|c|c|}
\hline & & \multicolumn{2}{|c|}{ Efficient blade } & \multicolumn{2}{|c|}{ Damaged blade } \\
\hline & & calculations & simulation & calculations & simulation \\
\hline$\omega$ & {$[\mathrm{rad} / \mathrm{s}]$} & 5,181 & & 7,464 & \\
\hline$f$ & {$[\mathrm{~Hz}]$} & 825 & 833 & 1,188 & 1,201 \\
\hline
\end{tabular}

Table 1 The results of calculations of natural frequency and modal analysis in CAD.
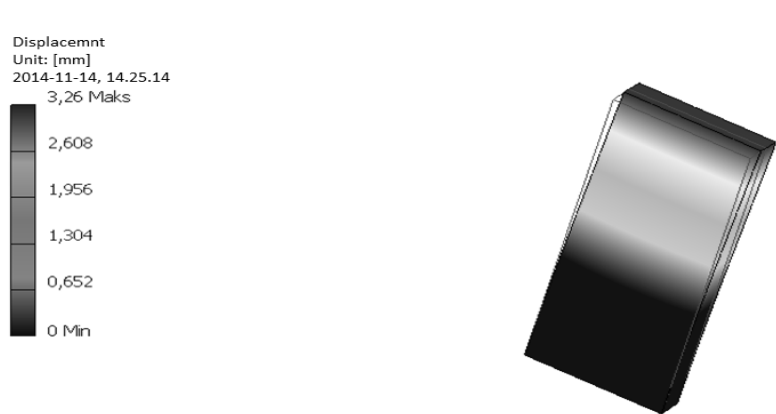

Fig. 1 Virtual modal analysis of efficient blade $(\mathbf{f 1}=$ 833.27 Hz).

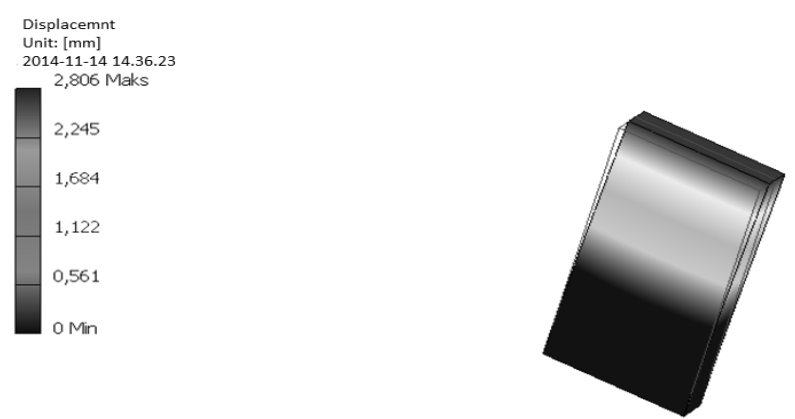

Fig. 2 Virtual modal analysis of damaged blade (f1 = $120,085 \mathrm{~Hz})$. 


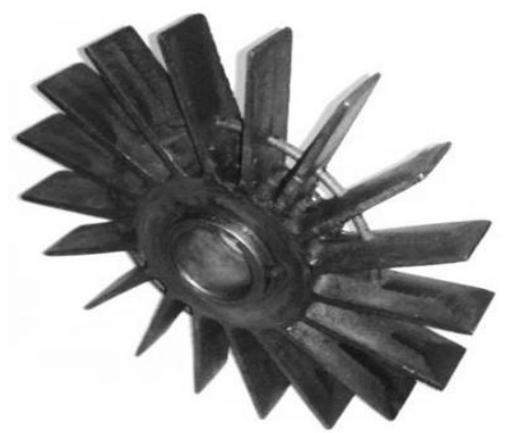

Fig. 3 SKA. 4.02 pump rotor-object of the investigations.

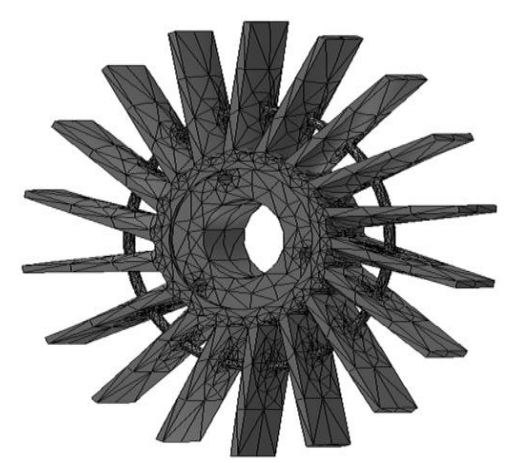

Fig. 4 Discretized model of rotor.

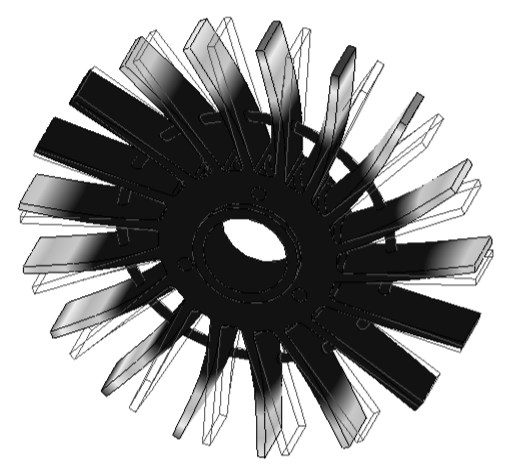

Fig. 5 An example of natural frequency mode.

of vibrations. EMA has many advantages. It is fast, relatively cheap and simple way to determine the resonance frequencies. The main equation of modal analysis is the general equation of the oscillating motion [3-5] (4):

$$
m \ddot{x}(t)+c \dot{x}(t)+k x(t)=f(t)
$$

where: $m$-mass, $c$ - damping factor,

$k$ - coefficient of elasticity.

Eq. (4) might be transferred to (5):

$$
\left[-m \omega^{2}+j c \omega+k\right] X(\omega)=F(\omega)
$$

Taking into account that:

$$
H(\omega)=\frac{1}{-m \omega^{2}+j c \omega+k}
$$

It might be wrote as:

$$
H(\omega)=\frac{X(\omega)}{F(\omega)}
$$

Value $H(\omega)$ is known as the frequency response function (FRF). FRF describes the Fourier transform value at the output of the $X(\omega)$ to the Fourier transform of input function working on particular system $F(\omega)$. When determining the value of FRF there are three types of estimators $H 1, H 2$ and $H 3$. Using the appropriate estimator depends on the expected components of the input and output [5]. The authors used the estimator $H 3$, suitable when the noise in the two signals is at a similar level. Application of $H 3$ estimator can minimize the influence of the noise of the input and output signal. $\mathrm{H3}$ estimator is given by Eq. (8) [4, 6-9]:

$$
H 3=\sqrt{H 1 \cdot H 2}
$$

$\mathrm{HI}$ estimator is used in the cases where the output signal has a high signal to noise ratio (SNR), H2 estimator is used to minimize the effect of noise in input signal. Estimators $\mathrm{HI}$ and $\mathrm{H} 2$ are describe by the Eqs. (9) and (10).

Table 2 Results of modal analysis carried out in CAD.

\begin{tabular}{llll}
\hline \multirow{2}{*}{ Mode } & Efficent rotor & Rotor with damaged blade & Rotor without blade \\
\cline { 2 - 4 } & $\mathrm{Hz}$ & $\mathrm{Hz}$ & $\mathrm{Hz}$ \\
\hline 1 & $1,938.67$ & $1,914.15$ & $1,793.77$ \\
2 & $1,939.63$ & $1,935.00$ & $1,903.95$ \\
3 & $2,182.28$ & $2,158.83$ & $2,036.50$ \\
4 & $2,182.31$ & $2,160.89$ & $2,158.67$ \\
5 & $2,331.78$ & $2,305.00$ & $2,240.75$ \\
6 & $2,379.00$ & $2,315.84$ & $2,347.53$ \\
7 & $2,423.79$ & $2,428.94$ & $2,347.74$ \\
\hline
\end{tabular}




$$
H 1=\frac{G_{X F}}{G_{X X}}
$$

$H 1$ estimator should be interpreted as the ratio of crosspectrum of output and input signal to the autospectrum of input signal [4].

$$
H 2=\frac{G_{F F}}{G_{X F}}
$$

$H 2$ estimator should be interpreted as the ratio of output signal autospectrum to the crosspectrum of the input and output. More detailed information on the issues are presented in $[4,6,8,10]$.

Discussing the problems related to the experimental modal analysis the coherence function cannot be ignored. Eq. (11) is a measure of cohesion and its value is comprised in the range of 0 to 1 :

$$
\gamma_{X F}^{2}=\frac{\left|G_{X F}\right|^{2}}{G_{X X} G_{F F}}
$$

Considering EMA, if coherence between the output and the input signals is 1 then it is certain that the system response was induced only by the recorded input signal. The coherence value less than 1 indicate presence of noise of unknown origin, however, such a signal can also be used during the further analysis of the EMA. However, it should be remembered to increase the amount of averaging. If the value of coherence is less than 0.5 then the EMA measurement should be carried out again.

\section{Results and Discussion}

Measurements were conducted with the system consisting of a hammer with a weight of $150 \mathrm{~g}$ with installed accelerometer. The same types accelerometers were installed in two mutually perpendicular directions on the rotor (Fig. 6). All signals were synchronized using a five channel $B \& K$ 3,650-B-120 front-end. All accelerometers were calibrated before and after the measurements using accelerometer calibrator type 4,294. During the measurements an accelerations of vibration were recorded in the band from $0.7 \mathrm{~Hz}$ to $12.8 \mathrm{kHz}$. It should be emphasized that, as a non-standard solution was used to excite the input signal. Commercially available modal hammers are equipped with force sensors, authors used piezoelectric acceleration sensor.

Analysis of the Eqs. (5)-(11) indicated that the applied solution will bring the desired results.

During the measurements accelerations in time domain were recorded. Signals of response and excitation were presented on Figs. 7 and 8. A series

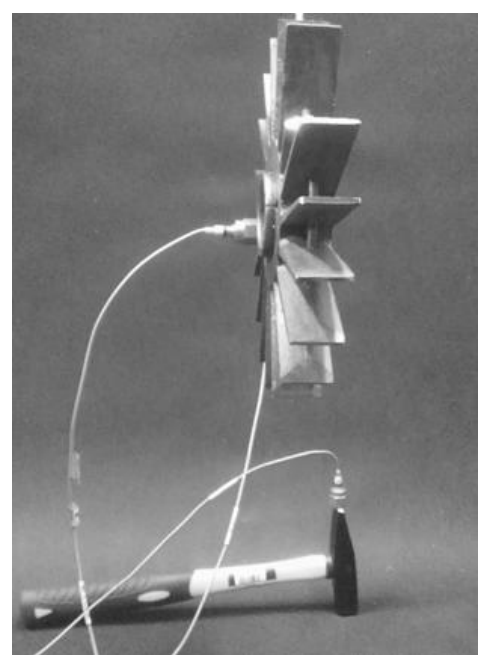

Fig. 6 Part of measurement set.

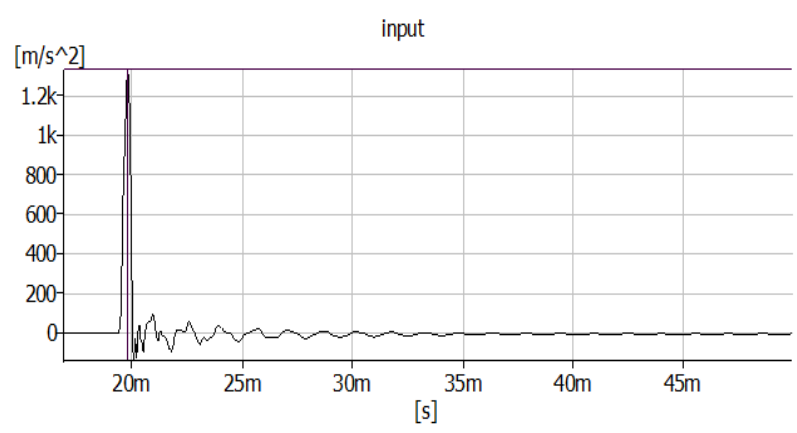

Fig. 7 An example of input signal obtained during measurement.

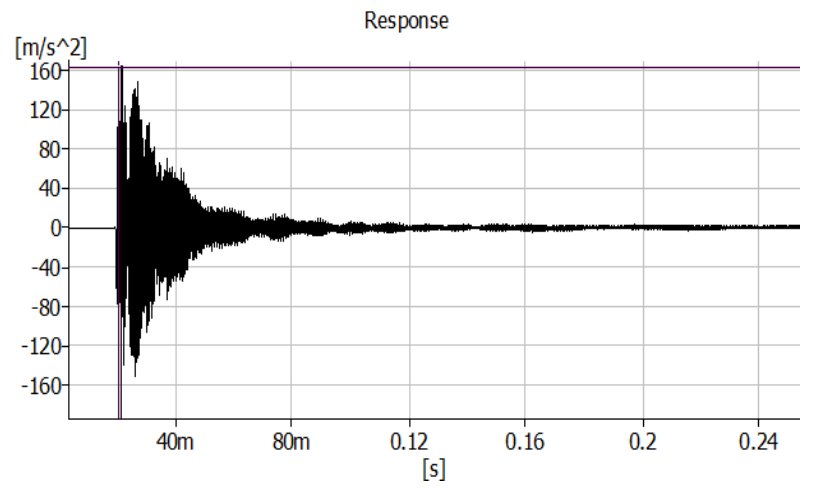

Fig. 8 An example of output signal recorded on rotor. 
Table 3 Summary of the resonant frequency measurements recorded during measurements.

\begin{tabular}{|c|c|c|c|c|c|}
\hline \multicolumn{2}{|c|}{ Undamaged rotor } & \multicolumn{2}{|c|}{ Rotor with damaged blade } & \multicolumn{2}{|c|}{ Rotor without blade } \\
\hline Frequency $[\mathrm{Hz}]$ & Coherence & Frequency $[\mathrm{Hz}]$ & Coherence & Frequency $[\mathrm{Hz}]$ & Coherence \\
\hline 2,074 & 0.999 & 2,099 & 0.998 & 1,936 & 0.991 \\
\hline 2,126 & 0.993 & 2,188 & 0.995 & 2,016 & 0.993 \\
\hline
\end{tabular}

Frequency Response H3(Signal 3,Signal 5) - Input (Magnitude) \FFT Analyzer $\left[\left(\mathrm{m} / \mathrm{s}^{\wedge} 2\right) /\left(\mathrm{m} / \mathrm{s}^{\wedge} 2\right)\right]$

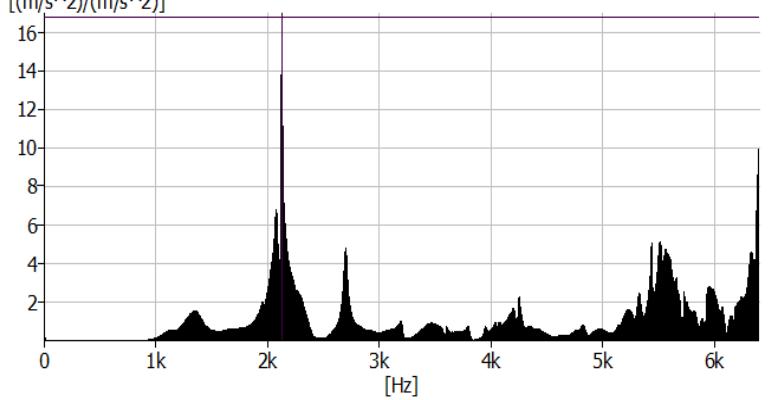

Fig. 9 Frequency response function between input and output obtained with $\mathrm{H3}$ estimator.

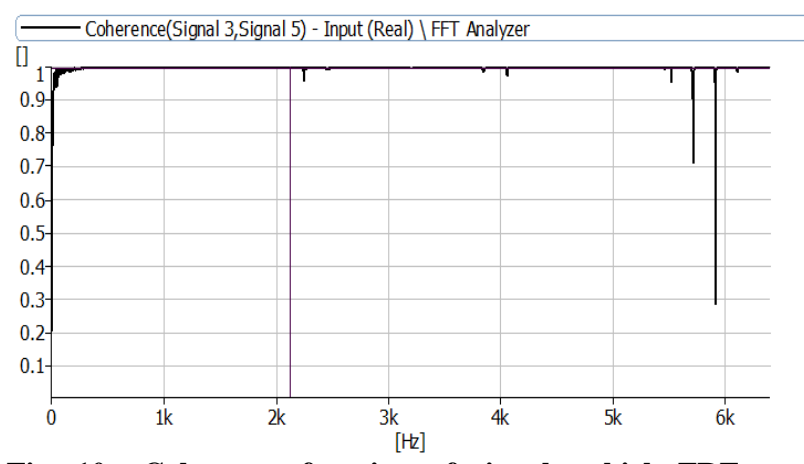

Fig. 10 Coherence function of signals which FRF was presented on picture 9.

of measurements were conducted and impact impulse was forced from different directions. Then, measurements were analyzed with Pulse Lab Shop software. First stage of signals selection was rejection of measurements with more than one high amplitude in input signal. The next step was to determine the frequency response function of the measurements. As a reference, input signal was always used. Frequency analysis due to the lack of significant amplitude at high frequencies was limited to $6.4 \mathrm{kHz}$. For further considerations only signals with coherency higher than 0.8 were taken. Final results of pump impeller experimental modal analysis were presented in Table 3. Examples of obtained frequency response function and coherence function were presented on Figs 9 and 10.
During the measurements analogous procedure to that used during the modeling procedure was used. Firstly, signals for efficient rotor were taken. The second step was the failure of one of the blades simulated by the cutting off a $0.03 \mathrm{~m}$ of one of them. Finally rotor without one blade was investigated.

\section{Conclusions}

Model tests as well as their experimental verification confirm a significant influence of damage of the rotor on its resonance frequency. Table 3 presents only the first two resonance frequencies obtained during measurements. They are close to the values gained during the analysis of the discrete model. It confirms the usefulness of the applied method of experimental modal analysis.

It should be noted that the method of simulating the weight loss does not occur in fact. Cavitation never damage only one blade of the rotor without affecting the other. Accurate analysis of the effect of cavitation on weight loss and its consequences on the resonant frequency will be considered in future publications. However physical results of damage one or more blades are the same. The aim of the study was to prepare a methodology to diagnose centrifugal pump rotor using non-destructive methods. This task may be important not only in the shipbuilding industry but also in power, chemical and petrochemical industries.

\section{References}

[1] Czyż W. 1992. Mechanika ogólna, cz. IVa, Drgania mechaniczne - teoria drgań. Gdynia: AMW.

[2] Dooms D., Degrande D., De Roeck G., and Reynders E.2006. "Finite Element Modelling of a Silo Based on Experimental Modal Analysis." Engineering Structures 28 (4): 532-542.

[3] Deuszkiewicz P., Dobrociński S., Dziurdź J., Flis L., Grządziela A., and Pakowski R., et. al. 2009. 


\section{Damage on Their Natural Frequency Response}

"Diagnostyka Wibroakustyczna Okrętowych Turbinowych Silników Spalinowych.” Radom: Monograficzna seria, Wydawnic two Instytutu Problemów eksploatacji-PIB.

[4] Harris C.M., eds. 2002. Harris' shock and vibration handbook-5th edition. New York: Donnelley \& Sons Company.

[5] Dudziński M., Grządziela A., and Kluczyk M. 2014. "Resonance Frequency Identification in a Combustion Gas Generator Model of a Turbine Engine with Order Tracking Procedure." Scientific Journal of Polish Naval Academy 2 (197) 5-22.

[6] Dossin O. 1988. Structural Testing-Modal Analysis and Simulation. Denmark: Bruel \& Kajer.

[7] Gade S., and Herlufsen H. 1994. Digital Filter Technique versus FFT Technique for Damping Measurements. Denmark: Brüel \& Kjær.

[8] Gade S., Herlufsen H., and Konstantin-Hansen H. 1996. How to Determine the Modal Parameters of Simple Structures. Denmark: Brüel \& Kjær.

[9] Łazarz B., Peruń G. 2009. "Identification and Verification of Simulation Model of Gears Working in Circulating Power System.” Diagnostyka 4: 55-60.

[10] Łukasiewicz, M., Kałaczyński, T., and Żółtowski, B. 2012. "Application of the Operational Modal Analysis in Transmission Gearbox Technical State Identification." Diagnostyka 3 (63) 23-26.

[11] Żółtowski B. 2004. “Analiza Modalna Diagnozowaniu Przekładni Zębatej.” Diagnostyka 23: 97-101. 